\title{
A partial order where all monotone maps are definable
}

\author{
by
}

\author{
Martin Goldster n (Wien and Berlin) and \\ Saharon Shelah (Jerusalem)
}

\begin{abstract}
It is consistent that there is a partial order $(P, \leq)$ of size $\aleph_{1}$ such that every monotone function $f: P \rightarrow P$ is first order definable in $(P, \leq)$.

It is an open problem whether there can be an infinite lattice $L$ such that every monotone function from $L$ to $L$ is a polynomial. Kaiser and Sauer [KS] showed that such a lattice would have to be bounded, and cannot be countable.
\end{abstract}

Sauer then asked the weaker question if there can be an infinite partial order $(P, \leq)$ such that all monotone maps from $P$ to $P$ are at least definable. (Throughout the paper, "definable" means "definable with parameters by a first order formula in the structure $(P, \leq)$ ".)

Since every infinite partial order $P$ admits at least $\mathfrak{c}=2^{\aleph_{0}}$ many monotone maps from $P$ to $P$, our partial order must have size (at least) continuum.

We show:

0.1. TheOREM. The statement "There is a partial order $(P, \leq)$ of size $\aleph_{1}$ such that all monotone functions $f: P \rightarrow P$ are definable in $P$ " is consistent relative to ZFC. Moreover, the statement holds in any model obtained by adding (iteratively) $\omega_{1}$ Cohen reals to a model of $\mathrm{CH}$.

We do not know if Sauer's question can be answered outright (i.e., in $\mathrm{ZFC}$ ), or even from $\mathrm{CH}$.

Structure of the paper. In Section 1 we give four conditions on a partial order on $(P, \sqsubseteq)$ of size $\kappa$ and we show that they are sufficient to ensure the 06А06.

1991 Mathematics Subject Classification: Primary 03C30; Secondary 03E35, 03C50,

This is number 554 in the second author's publication list. Supported by the Israeli Academy of Sciences. 
conclusion of the theorem. This section is very elementary. The two main conditions of Section 1 are

(1) a requirement on small sets, namely that they should be definable,

(2) two requirements on large sets (among them: "there are no large antichains").

Here, "small" means of size $<|P|$, and "large" means of size $=|P|$.

In Section 2 we show how to take care of requirement (1) in an inductive construction of our partial order in $\mathfrak{c}$ many steps. Each definability requirement will be satisfied at some stage $\alpha<\mathfrak{c}$.

Finally, in Section 4 we deal with the problem of avoiding large antichains. Here the inductive construction is not so straightforward, as we have to "anticipate" potential large sets and ensure that in the end they will not contradict our requirement. The standard tool for dealing with such a problem is $\diamond$. This combinatorial principle has been used for a related construction in [Sh 128], and it is possible to use the techniques of [Sh 136, Section 5] to combine it with the requirement on small sets to show that the conclusion of our theorem actually follows from $\diamond$. However, we use instead a forcing construction, which seems to be somewhat simpler: We will work in an iterated forcing extension, and use a $\Delta$-system argument to ensure that the requirements about large sets are met. This argument is carried out in Section 4, which therefore requires a basic knowledge of forcing.

\section{Four conditions}

1.1. Theorem. Assume that $(P, \sqsubseteq)$ is a partial order, and $\kappa:=|P| a$ regular cardinal. We will call subsets of cardinality $<\kappa$ "small". Assume that the following conditions hold:

C1. Every antichain is small (an antichain is a set of pairwise incomparable elements).

C2. Whenever $g:(P, \sqsubseteq) \rightarrow(P, \sqsubseteq)$ is monotone, then there is a small set $A \subseteq P$ such that for all $\alpha \in P, f(\alpha) \in A \cup\{\alpha\}$.

C3. For all $\alpha \in P$ the set $\{\beta \in P: \beta \sqsubseteq \alpha\}$ is small.

C4. Every small subset of $P \times P$ is definable in the structure $(P, \sqsubseteq)$.

Then every monotone map from $(P, \sqsubseteq)$ to itself is definable in $(P, \sqsubseteq)$.

We will prove this theorem below.

Lemma 1.2. Let $\sqsubseteq$ be as above. Then for any set $A \subseteq P$ there is a small set $A^{\prime} \subseteq A$ such that $\forall \alpha \in A \exists \gamma \in A^{\prime}: \gamma \sqsubseteq \alpha$.

Pr o of. Let $B \subseteq A$ be a maximal antichain in $A$. So $B$ is small, by $\mathrm{C} 1$. Let $A^{\prime}$ be the downward closure of $B$ in $A$, i.e., $A^{\prime}:=\bigcup_{\beta \in B}\{\gamma \in A: \gamma \sqsubseteq \beta\}$. Then $A^{\prime}$ is still small because of C3. Clearly $A^{\prime}$ is as required. 
1.3. FACT. Let $(P, \sqsubseteq)$ be any partial order, and $g: P \rightarrow P$ a monotone function. Then $g$ is definable iff the set $\{(x, y): g(x) \sqsubseteq y\}$ is definable.

Proof. Let $B:=\{(x, y): g(x) \sqsubseteq y\}$. Clearly $B$ is definable from $g$. Conversely, $g$ can be defined from $B$ as follows:

$$
g(x)=z \Leftrightarrow(\forall y:(x, y) \in B \Rightarrow z \sqsubseteq y) \text { and }((x, z) \in B) .
$$

Pro of of 1.1. Let $g: P \rightarrow P$ be monotone. Let $P_{0} \subseteq P$ be small such that

- If $g(\alpha) \neq \alpha$ then $g(\alpha) \in P_{0}$.

- $\beta \sqsubseteq \alpha \in P_{0}$ implies $\beta \in P_{0}$.

(Such a set $P_{0}$ can be found using $\mathrm{C} 2$ and C3.) For every $j \in P_{0}$ let $D_{j}:=$ $\left\{\alpha \in P \backslash P_{0}: g(\alpha)=j\right\}$, and let $D:=\left\{\alpha \in P \backslash P_{0}: g(\alpha)=\alpha\right\}$. We can find $D^{\prime}$ and $D_{j}^{\prime}$ as in 1.2. Let $P_{1}=\bigcup_{j} D_{j}^{\prime} \cup D^{\prime}$.

Claim 1. For $\alpha \in P \backslash P_{0}$ we have

$$
g(\alpha)=\alpha \Leftrightarrow \exists \beta \in P_{1}: \beta \sqsubseteq \alpha, g(\beta)=\beta .
$$

The direction $\Rightarrow$ follows immediately from the definition of $D^{\prime}$. For the other direction, assume that $\beta \in P_{1}, \beta \sqsubseteq \alpha, g(\beta)=\beta$. Since $g(\beta) \sqsubseteq g(\alpha)$, and $g(\beta)=\beta \notin P_{0}$, we also have $g(\alpha) \notin P_{0}$. Hence $g(\alpha)=\alpha$.

Claim 2. For $\alpha \in P \backslash P_{0}, g(\alpha) \neq \alpha$ we have

$$
g(\alpha) \sqsubseteq i \Leftrightarrow \forall \gamma \in P_{1}: \gamma \sqsubseteq \alpha \Rightarrow g(\gamma) \sqsubseteq i .
$$

The implication $\Rightarrow$ follows from the monotonicity of $g$. For the converse direction, assume that $g(\alpha)=j$. Since $D_{j}^{\prime} \subseteq P_{1}$, we can find $\gamma \in P_{1}$ with $\gamma \sqsubseteq \alpha$ and $g(\gamma)=j$. By assumption, $g(\gamma) \sqsubseteq i$, so $g(\alpha) \sqsubseteq i$.

Claims 1 and 2, together with 1.3, now imply that $g$ can be defined from the graphs of the functions $g \nmid P_{0}$ and $g\left\lceil P_{1}\right.$.

2. Coding small sets. In this section we will show how to build a partial order satisfying C3 and C4. Throughout this section we assume the continuum hypothesis.

2.1. Definition. (1) We call $\mathfrak{M}$ a creature if $\mathfrak{M}=(M, \sqsubseteq, F, H)$, where

- $\sqsubseteq$ is a partial order on $M$,

- $F$ is a partial symmetric function, $\operatorname{dom}(F) \subseteq M \times M, \operatorname{ran}(F) \subseteq M$,

- if $F(x, y)=z$, then $x<z, y<z$, and there is no $z^{\prime} \neq z$ with $x \sqsubseteq z^{\prime}$, $y \sqsubseteq z^{\prime}, z^{\prime} \sqsubseteq z$ (i.e. $z$ is a minimal upper bound of $x$ and $y$, and $x, y$ are incomparable),

- $F$ is locally finite, i.e., for any finite $A \subseteq M$ there is a finite set $B$ with $A \subseteq B \subseteq M$ such that for any $(x, y) \in \operatorname{dom} F \cap(B \times B), F(x, y) \in B$,

- $H \subseteq M \times M \times M$; we define $H(x, y):=\{z: H(x, y, z)\}$, 
- if $H(x, y, z)$ then $z$ is a minimal upper bound of $x, y$, and $x, y$ are incomparable,

- $H(x, y)=H(y, x)$,

- if $H(x, y, z)$ then $(x, y) \notin \operatorname{dom}(F)$.

(2) If $\mathfrak{M}_{1}, \mathfrak{M}_{2}$ are creatures, then $\mathfrak{M}_{1} \leq \mathfrak{M}_{2}$ ( $\mathfrak{M}_{2}$ is an extension of $\mathfrak{M}_{1}$ ) means that $M_{1} \subseteq M_{2}$, and the relations/functions of $\mathfrak{M}_{1}$ are just the restrictions of the corresponding relations/functions of $\mathfrak{M}_{2}$ (so in particular, $\left.\operatorname{dom}\left(F^{\mathfrak{M}_{2}}\right) \cap\left(M_{1} \times M_{2}\right)=\operatorname{dom}\left(F^{\mathfrak{M}_{1}}\right)\right)$.

(3) We say that $\mathfrak{M}_{2}$ is an end extension of $\mathfrak{M}_{1}$ (or that $\mathfrak{M}_{1}$ is an initial segment of $\left.\mathfrak{M}_{2}\right)$ if $\mathfrak{M}_{1} \leq \mathfrak{M}_{2}$ and $\mathfrak{M}_{1}$ is downward closed in $\mathfrak{M}_{2}$, i.e., $\mathfrak{M}_{2} \models$ $x \sqsubseteq y, y \in M_{1}$ implies $x \in M_{1}$.

(4) We use the above terminology also if one or both of the structures $\mathfrak{M}_{1}$ $\mathfrak{M}_{2}$ are just partial orders without an additional function $F$ or relation $H$.

2.2. Notation. When we consider several creatures $\mathfrak{M}, \mathfrak{M}_{1}, \mathfrak{M}^{*}, \ldots$, then it is understood that their universes are called $M, M_{1}, M^{*}, \ldots$, their partial orders are $\sqsubseteq, \sqsubseteq_{1}, \sqsubseteq^{*}, \ldots$, etc. Instead of writing $x \sqsubseteq_{1} y$ we may write $\mathfrak{M}_{1}=x \sqsubseteq y$, etc.

We will use the letters $\mathfrak{M}$ and $\mathfrak{N}$ to denote possibly infinite creatures, and $\mathfrak{p}, \mathfrak{q}$ to denote finite creatures.

2.3. Definition. If $(M, \sqsubseteq)$ is a partial order, then we let $\mathcal{F}(\sqsubseteq)$ be the partial binary function $F$ satisfying

$$
F(x, y)=\left\{\begin{aligned}
\text { the unique } & \text { minimal upper bound of } x \text { and } y \\
& \text { if it exists and if } x \text { and } y \text { are incomparable } \\
\text { undefined } & \text { otherwise, }
\end{aligned}\right.
$$

2.4. Setup. Let $\left(M_{\delta}: \delta<\mathfrak{c}\right)$ be a continuous increasing sequence of infinite sets with $\left|M_{\delta+1} \backslash M_{\delta}\right|=\left|M_{\delta}\right|<\mathfrak{c}$. Let $\left(R_{\delta}: \delta<\mathfrak{c}\right)$ be a sequence of relations with $R_{\delta} \subseteq M_{\delta} \times M_{\delta}$ such that for any $R \subseteq M_{\delta} \times M_{\delta}$ there is a $\delta^{\prime}>\delta$ such that $R=R_{\delta^{\prime}}$. (Such a sequence can be found using CH.)

For each $\delta$ we fix some element $e_{\delta} \in M_{\delta+1} \backslash M_{\delta}$. For each $(\alpha, \beta) \in M_{\delta} \times M_{\delta}$ we pick disjoint sets $A_{\alpha \beta}^{\delta}, B_{\alpha \beta}^{\delta}, C_{\alpha \beta}^{\delta}, \Delta_{\alpha \beta}^{\delta}, \Gamma_{\alpha \beta}^{\delta}$ (we will omit the superscript $\delta$ if it is clear from the context) satisfying

$$
\begin{gathered}
\left|A_{\alpha \beta}\right|=2, \quad\left|B_{\alpha \beta}\right|=3, \quad\left|C_{\alpha \beta}\right|=1, \\
\left|\Delta_{\alpha \beta}\right|=3, \quad \Delta_{\alpha \beta}=\left\{a_{\alpha \beta}, b_{\alpha \beta}, c_{\alpha \beta}\right\}, \quad \Gamma_{\alpha \beta}=\left\{\gamma_{\alpha \beta}\right\},
\end{gathered}
$$

where $e_{\delta}$ is not in any of these sets and the sets

$$
\Omega_{\alpha \beta}:=A_{\alpha \beta} \cup B_{\alpha \beta} \cup C_{\alpha \beta} \cup \Delta_{\alpha \beta} \cup \Gamma_{\alpha \beta}
$$

satisfy $(\alpha, \beta) \neq\left(\alpha^{\prime}, \beta^{\prime}\right) \Rightarrow \Omega_{\alpha \beta} \cap \Omega_{\alpha^{\prime} \beta^{\prime}}=\emptyset$. For $x \in \Omega_{\alpha \beta}$ we define $\Omega(x):=$ $\Omega_{\alpha \beta} \cup\{\alpha, \beta\}$.

Moreover, we choose the sets $\Omega_{\alpha \beta}$ such that $M_{\delta+1} \backslash \bigcup_{\alpha, \beta \in M_{\delta}} \Omega_{\alpha \beta}$ still has size $\left|M_{\delta}\right|$. For $x \in M_{\delta+1} \backslash \bigcup_{\alpha, \beta \in M_{\delta}} \Omega_{\alpha \beta}$ we let $\Omega(x)=\emptyset$. 
2.5. Definition. (1) Let $\mathfrak{M}$ be a creature. We say that $\Delta \in[M]^{3}$ is a triangle (also called an $\mathfrak{M}$-triangle or $F$-triangle if $F=F^{\mathfrak{M}}$ ) iff $\operatorname{dom}\left(F^{\mathfrak{M}}\right.$ ) $\supseteq[\Delta]^{2}$.

(2) Let $\Delta$ be an $F$-triangle. We say that $a \in \Delta$ is a base point for $\Delta$ if there is a unique (unordered) pair $\{b, c\}$ with $F(b, c)=a$.

(3) Let $\Delta$ be a triangle. We say that $b$ is an anchor for $\Delta$ if there is a $c$ such that $F(b, c)$ is a base point for $\Delta$.

(4) If $\mathfrak{M}_{1} \leq \mathfrak{M}_{2}$ then we say that $\mathfrak{M}_{2}$ is a separated extension of $\mathfrak{M}_{1}$ iff

- every triangle in $\mathfrak{M}_{2}$ is contained in $M_{1}$ or in $M_{2} \backslash M_{1}$, and

- if $\Delta \subseteq M_{2} \backslash M_{1}$ is a triangle, then $\Delta$ is not anchored at any point in $M_{1}$.

- if $\mathfrak{M}_{2}=H(x, y, z)$ and $x, y \in M_{1}$, then also $z \in M_{1}$.

2.6. Note. Our goal is to define $F_{\delta+1}$ such that $R_{\delta}$ will become definable (and at the same time make it possible for $F_{\delta+1}$ to be of the form $\mathcal{F}(\sqsubseteq)$ for some end extension $\sqsubseteq$ of $\left.\sqsubseteq_{\delta}\right)$. We will achieve this by "attaching" triangles to all pairs $(\alpha, \beta)$ in $R_{\delta}$ in a way that $(\alpha, \beta)$ can be reconstructed from the triangle. We also need to ensure that the only triangles in $\mathfrak{M}_{\delta+1}$ (and also in any $\mathfrak{M}_{\delta^{\prime}}, \delta^{\prime}>\delta$ ) are the ones we explicitly put there.

The particular way of coding pairs by triangles is rather arbitrary.

2.7. Overview of the construction. By induction on $\delta \leq \mathfrak{c}$ we will define creatures $\mathfrak{M}_{\delta}=\left(M_{\delta}, \sqsubseteq_{\delta}, F_{\delta}\right)$ such that

(A) $\gamma<\delta$ implies that $\mathfrak{M}_{\delta}$ is a separated end extension of $\mathfrak{M}_{\gamma}$,

(B) $F_{\delta}=\mathcal{F}\left(\sqsubseteq_{\delta}\right)($ see 2.3$)$,

(C) $R_{\delta}$ is definable in $\left(M_{\delta+1}, \sqsubseteq_{\delta+1}, F_{\delta+1}\right)$ and hence also in $\left(M_{\delta+1}, \sqsubseteq_{\delta+1}\right)$, and the definition of $R_{\delta}$ is absolute for any separated end extension of $\mathfrak{M}_{\delta+1}$.

For limit $\delta$ we let $\sqsubseteq_{\delta}=\bigcup_{\gamma<\delta} \sqsubseteq_{\gamma}$ and $F_{\delta}=\bigcup_{\gamma<\delta} F_{\gamma}, H_{\delta}=\bigcup_{\gamma<\delta} H_{\gamma}$.

We will construct $\mathfrak{M}_{\delta+1}$ from $\mathfrak{M}_{\delta}$ in two steps. First we define a function $F_{\delta+1}$ such that $R_{\delta}$ becomes definable in $\left(M_{\delta+1}, F_{\delta+1}\right)$. Then we show that we can find a partial order $\sqsubseteq_{\delta+1}$ (end-extending $\sqsubseteq_{\delta}$ ) such that $F_{\delta+1}$ $=\mathcal{F}\left(\sqsubseteq_{\delta+1}\right)$.

Construction of $F_{\delta+1} \cdot F_{\delta+1}$ will be defined as follows:

- $F_{\delta+1} \uparrow\left(M_{\delta} \times M_{\delta}\right)=F_{\delta}$.

- If $(\alpha, \beta) \in R_{\delta}$ then

$* F(\alpha, x)=a_{\alpha \beta}$ for $x \in A_{\alpha \beta}$,

$* F(\beta, x)=b_{\alpha \beta}$ for $x \in B_{\alpha \beta}$,

$* F\left(e_{\delta}, x\right)=c_{\alpha \beta}$ for $x \in C_{\alpha \beta}$,

$* F(x, y)=\gamma_{\alpha \beta}$ for $\{x, y\} \in\left[\Delta_{\alpha \beta}\right]^{2}$.

- Except where required by the above (and by symmetry), $F_{\delta+1}$ is undefined. 
- $\gamma_{\alpha \beta}$

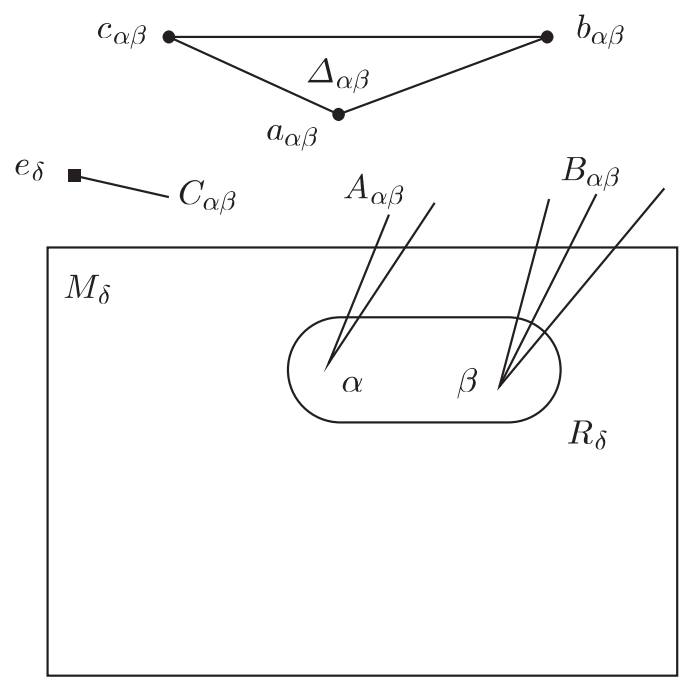

This completes the definition of $F_{\delta+1}$. The diagram above is supposed to illustrate this definition. Pairs $(x, y)$ on which $F_{\delta+1}$ is defined are connected by a line; the value of $F_{\delta+1}$ at such pairs is the small black disk above the pair.

2.8. FACT. (1) $\left(M_{\delta+1}, F_{\delta+1}\right)$ is a separated extension of $\mathfrak{M}_{\delta}$.

(2) $R_{\delta}$ is definable in $\left(M_{\delta+1}, F_{\delta+1}\right)$.

(3) Let $\left(M_{\delta+1}, F_{\delta+1}\right) \leq(M, F)$ and assume that

- $(M, F)$ is a separated extension of $\left(M_{\delta+1}, F_{\delta+1}\right)$, and

- if $F(x, y) \in M_{\delta+1}$ then $x, y \in M_{\delta+1}$. (This is certainly true if $F=\mathcal{F}(\sqsubseteq)$, where $\sqsubseteq$ is an end extension of $\left.\sqsubseteq_{\delta+1}.\right)$

Then $R_{\delta}$ is definable in $(M, F)$.

Proof. (1) is clear. (2) is a special case of (3). Let $(M, F)$ be as in (3). Then $R_{\delta}$ is the set of all pairs $(\alpha, \beta)$ such that there is a triangle $\Delta$ with a unique base, anchored at $e_{\delta}$ such that either

- $\alpha \neq \beta$ and $|\{x: F(\alpha, x) \in \Delta\}|=2,|\{x: F(\beta, x) \in \Delta\}|=3$, or

- $\alpha=\beta$ and $|\{x: F(\alpha, x) \in \Delta\}|=5$,

because the only triangles anchored at $e_{\delta}$ will be the sets $\Delta_{\alpha \beta}^{\delta}$. Clearly this is a definition in first order logic with the parameter $e_{\delta}$.

Construction of $\sqsubseteq_{\delta+1}$. We will define $\sqsubseteq_{\delta+1}$ from a (sufficiently) generic filter for a forcing notion $\mathbb{Q}_{\delta}=\mathbb{Q}\left(\mathfrak{M}_{\delta}, F_{\delta+1}\right)$. 
2.9. Definition. Assume that $\mathfrak{M}_{\delta}, F_{\delta+1}$ are as above. We define the forcing notion $\mathbb{Q}_{\delta}$ as the set of all $\mathfrak{p}$ such that

- $\mathfrak{p}$ is a finite creature, $\mathfrak{p}=\left(p, \sqsubseteq_{p}, F_{p}, H_{p}\right)$,

$\bullet \mathfrak{p}\left\lceil M_{\delta} \leq \mathfrak{M}_{\delta}\right.$

- $p \subseteq M_{\delta+1}$,

- for all $x \in p \backslash M_{\delta}, \Omega(x) \subseteq p$,

- $F_{p}=F_{\delta+1} \uparrow(p \times p)$,

- $\mathfrak{p}$ is a separated end extension of $\mathfrak{p}\left\lceil M_{\delta}\right.$.

Clearly $\left(\mathbb{Q}_{\delta}, \leq\right)$ is a partial order. N ot e: We force "upwards," i.e., $\mathfrak{p} \leq \mathfrak{q}$ means that $\mathfrak{q}$ is a stronger condition than $\mathfrak{p}$. But we still call the generic set "filter" and not "ideal".

If $G \subseteq Q_{\delta}$ is a filter then there is a smallest creature which extends all $\mathfrak{p} \in G$. We call this creature $\mathfrak{M}_{G}$.

2.10. FACT. The following sets are dense in $\mathbb{Q}_{\delta}$ :

(a) $\{\mathfrak{p}: x \in p\}$, for any $x \in M_{\delta+1}$,

(b) $D_{\mathfrak{p}, x, y}:=\left\{\mathfrak{q}: \mathfrak{q} \perp \mathfrak{p}\right.$ or $\left.\mathfrak{q}=\exists z^{\prime} \notin p: H(x, y, z)\right\}$, whenever $x, y \in p$ are incomparable and $(x, y) \notin \operatorname{dom}\left(F_{\mathfrak{p}}\right)$,

(c) $E_{\mathfrak{p}, x, y, z}:=\left\{\mathfrak{q}: \mathfrak{q} \perp \mathfrak{p}\right.$ or $\left.\mathfrak{q} \models \exists z^{\prime} \sqsubseteq z: x \sqsubseteq z^{\prime}, y \sqsubseteq z^{\prime}, z \neq z^{\prime}\right\}$, whenever $x, y \in p,(x, y) \in \operatorname{dom}\left(F_{\mathfrak{p}}\right), x \sqsubseteq z, y \sqsubseteq z, z \neq F_{\mathfrak{p}}(x, y)$.

2.11. FACT. If $G$ meets all the dense sets above, then $\mathfrak{M}_{\delta+1}:=\mathfrak{M}_{G}$ is a separated end extension of $\mathfrak{M}_{\delta}$, and $F_{\delta+1}=\mathcal{F}\left(\sqsubseteq_{\mathfrak{M}_{G}}\right)$.

Proof. We only prove the last statement. Let $F=\mathcal{F}\left(\Xi_{\mathfrak{M}_{G}}\right)$. First assume $F_{\delta+1}(x, y)=z^{*}$. Clearly $z^{*}$ is a minimal upper bound of $x, y$, so to prove $F(x, y)=z^{*}$ we only have to show that $z^{*}$ is the unique minimal upper bound. If $z \neq z^{*}$ is also a minimal upper bound then we can find $p \in G$ containing $\left\{x, y, z, z^{*}\right\}$. Now use $2.10(\mathrm{c})$ to find $z^{\prime} \neq z$ in $M_{\delta+1}$, $x, y \sqsubseteq z^{\prime} \sqsubseteq z$, which is a contradiction.

Now assume that $F_{\delta+1}(x, y)$ is undefined. We have to show that also $F(x, y)$ is undefined. Applying 2.10(b) twice we can find two distinct elements $z_{1}, z_{2}$ such that $H\left(x, y, z_{1}\right)$ and $H\left(x, y, z_{2}\right)$ both hold in $\mathfrak{M}_{\delta+1}$, hence there is no unique minimal upper bound of $x$ and $y$, so $F(x, y)$ is undefined.

2.12. FACT. $\mathbb{Q}_{\delta}$ satisfies the ccc.

Proof. $Q_{\delta}$ is countable.

2.13. Conclusion. CH implies that a sufficiently generic filter exists. Thus we have completed the definition of $\sqsubseteq_{\delta+1}$ and $H_{\delta+1}$. Clearly $\mathfrak{M}_{\delta+1}$ will be as required. 
In the last section we will show how to embed this construction into an iterated forcing argument.

3. Amalgamation. Starting from a model of GCH we will construct an iterated forcing notion $\left(\mathbb{P}_{\delta}, Q_{\delta}: \delta<\kappa\right)$ such that each partial order $Q_{\delta}$ will be some $\mathbb{Q}\left(\mathfrak{M}_{\delta}, F_{\delta+1}\right)$ as in the previous section. This will define a model $\mathfrak{M}_{\kappa}$. An additional argument is then needed to show that $\mathfrak{M}_{\kappa}$ will satisfy $\mathrm{C} 1$ and $\mathrm{C} 2$. In this section we prepare some tools for this additional argument by collecting some facts about amalgamation.

3.1. Definition. Let $\mathfrak{p}$ and $\mathfrak{q}$ be (finite) creatures, $x \in p, y \in q$. We define $\mathfrak{p} \oplus \mathfrak{q}$ and $\mathfrak{p} \oplus_{x, y} \mathfrak{q}$ as follows:

- $\mathfrak{p} \oplus \mathfrak{q}=\left(p \cup q, \sqsubseteq_{\mathfrak{p} \oplus \mathfrak{q}}, F_{\mathfrak{p} \oplus \mathfrak{q}}, H_{\mathfrak{p} \oplus \mathfrak{q}}\right)$, where $\sqsubseteq_{\mathfrak{p} \oplus \mathfrak{q}}$ is the transitive closure of $\sqsubseteq_{\mathfrak{p}} \cup \sqsubseteq_{\mathfrak{q}}$, and $F_{\mathfrak{p} \oplus \mathfrak{q}}=F_{\mathfrak{p}} \cup F_{\mathfrak{q}}$.

- $\mathfrak{p} \oplus_{x, y} \mathfrak{q}=\left(p \cup q, \sqsubseteq_{\mathfrak{p} \oplus_{x, y} \mathfrak{q}}, F_{\mathfrak{p} \oplus_{x, y} \mathfrak{q}}, H_{\mathfrak{p} \oplus_{x, y} \mathfrak{q}}\right)$, where $\sqsubseteq_{\mathfrak{p} \oplus_{x, y} \mathfrak{q}}$ is the transitive closure of $\sqsubseteq_{\mathfrak{p}} \cup \sqsubseteq_{\mathfrak{q}} \cup\{(x, y)\}$, and $F_{\mathfrak{p} \oplus_{x, y} \mathfrak{q}}=F_{\mathfrak{p}} \cup F_{\mathfrak{q}}, H_{\mathfrak{p} \oplus_{x, y} \mathfrak{q}}=H_{\mathfrak{p}} \cup H_{\mathfrak{q}}$.

3.2. FACT. (1) Assume that $\sqsubseteq_{\mathfrak{p}}$ and $\sqsubseteq_{\mathfrak{q}}$ agree on $p \cap q$, and similarly $F_{\mathfrak{p}}$ and $F_{\mathfrak{q}}$. Then $\mathfrak{p} \oplus \mathfrak{q}$ is a creature, and $\mathfrak{p} \sqsubseteq \mathfrak{p} \oplus \mathfrak{q}, \mathfrak{q} \sqsubseteq \mathfrak{p} \oplus \mathfrak{q}$.

(2) If $\mathfrak{p}$ and $\mathfrak{q}$ are as above, and moreover, $\mathfrak{p}$ and $\mathfrak{q}$ are separated end extensions of $\mathfrak{r}:=\mathfrak{p} \cap \mathfrak{q}$, and type $\mathrm{e}_{\mathfrak{p}}(x, \mathfrak{r})=\operatorname{type}_{\mathfrak{q}}(y, \mathfrak{r})$ (that is, for any $z \in r$ we have $\mathfrak{p}=z \sqsubseteq x$ iff $\mathfrak{q}=z \sqsubseteq y$ ), then also $\mathfrak{p} \oplus_{x, y} \mathfrak{q}$ is a model extending $\mathfrak{q}$ and end-extending $\mathfrak{p}$, and

(*) $\mathfrak{p} \oplus_{x, y} \mathfrak{q} \models a \sqsubseteq b \Leftrightarrow \mathfrak{p}=a \sqsubseteq b$ or $\mathfrak{q}=a \sqsubseteq b$ or $\mathfrak{p}=a \sqsubseteq x, \mathfrak{q} \models y \sqsubseteq b$.

Pro of. We leave (1) to the reader. Let $\sqsubseteq^{*}$ be the relation defined in $(*)$. First we have to check that $\complement^{*}$ is transitive. Note that the third clause in the definition of $a \sqsubseteq^{*} b$ can only apply if $a \in p$ and $b \in q \backslash r=q \backslash p$.

Let $a \sqsubseteq^{*} b \sqsubseteq^{*} c$. A priori, there are 9 possible cases:

$$
\begin{aligned}
& a \sqsubseteq_{\mathfrak{p}} b \quad b \sqsubseteq_{\mathfrak{p}} c \quad \Rightarrow a \sqsubseteq_{\mathfrak{p}} c \\
& a \sqsubseteq_{\mathfrak{p}} b \quad b \sqsubseteq_{\mathfrak{q}} c \quad \Rightarrow b \in p \cap q, a \sqsubseteq_{\mathfrak{q}} b, \text { so } a \sqsubseteq_{\mathfrak{q}} c \\
& a \sqsubseteq_{\mathfrak{p}} b \quad b \sqsubseteq_{\mathfrak{p}} x, y \sqsubseteq_{\mathfrak{q}} c \Rightarrow a \sqsubseteq_{\mathfrak{p}} x, y \sqsubseteq_{\mathfrak{q}} c \\
& a \sqsubseteq_{\mathfrak{q}} b \quad b \sqsubseteq_{\mathfrak{p}} c \quad \Rightarrow b \in p \cap q, a \sqsubseteq_{\mathfrak{p}} b \text {, so } a \sqsubseteq_{\mathfrak{p}} c \\
& a \sqsubseteq_{\mathfrak{q}} b \quad b \sqsubseteq_{\mathfrak{q}} c \quad \Rightarrow a \sqsubseteq_{\mathfrak{q}} c \\
& a \sqsubseteq_{\mathfrak{q}} b \quad b \sqsubseteq_{\mathfrak{p}} x, y \sqsubseteq_{\mathfrak{q}} c \Rightarrow b \in p \cap q, a \sqsubseteq_{\mathfrak{p}} b, a \sqsubseteq_{\mathfrak{p}} x, y \sqsubseteq_{\mathfrak{q}} c \\
& a \sqsubseteq_{\mathfrak{p}} x, y \sqsubseteq_{\mathfrak{q}} b \quad b \sqsubseteq_{\mathfrak{p}} c \quad \Rightarrow \text { impossible } \\
& a \sqsubseteq_{\mathfrak{p}} x, y \sqsubseteq_{\mathfrak{q}} b \quad b \sqsubseteq_{\mathfrak{q}} c \quad \Rightarrow a \sqsubseteq_{\mathfrak{p}} x, y \sqsubseteq_{\mathfrak{q}} c \\
& a \sqsubseteq_{\mathfrak{p}} x, y \sqsubseteq_{\mathfrak{q}} b \quad b \sqsubseteq_{\mathfrak{p}} x, y \sqsubseteq_{\mathfrak{q}} c \Rightarrow \text { impossible }
\end{aligned}
$$

So we see that in any case we get $a \sqsubseteq^{*} c$. Clearly $\mathfrak{p} \oplus_{x, y} \mathfrak{q}$ is an end extension of $\mathfrak{p}$. We now check that $\mathfrak{q} \leq \mathfrak{p} \oplus_{x, y} \mathfrak{q}$. Let $a, b \in q, \mathfrak{p} \oplus_{x, y} \mathfrak{q}=a \sqsubseteq b$. The only nontrivial case is where $\mathfrak{p} \models a \sqsubseteq x$ and $\mathfrak{q} \models y \leq b$. Since $x$ and $y$ 
have the same type over $\mathfrak{p} \cap \mathfrak{q}$, we also have $\mathfrak{q} \mid=a \sqsubseteq y$. Hence $\mathfrak{q} \models a \sqsubseteq b$, so $\mathfrak{q} \leq \mathfrak{p} \oplus_{x, y} \mathfrak{q}$.

Finally, we check that $\mathfrak{p} \oplus_{x, y} \mathfrak{q}$ is a creature. It is clear that $F^{\mathfrak{p} \oplus_{x, y} \mathfrak{q}}$ is locally finite.

To check that the conditions on $H_{\mathfrak{p} \oplus_{x, y} \mathfrak{q}}$ and $F_{\mathfrak{p} \oplus_{x, y} \mathfrak{q}}$ (in particular, minimality) are still satisfied, the following key fact is sufficient: if $\{a, b\} \subseteq p$ or $\{a, b\} \subseteq q$ (in particular, if $\left.(a, b) \in \operatorname{dom}\left(H_{\mathfrak{p} \oplus_{x, y} \mathfrak{q}}\right) \cup \operatorname{dom}\left(F_{\mathfrak{p} \oplus_{x, y} \mathfrak{q}}\right)\right)$, then $\mathfrak{p} \oplus_{x, y} \mathfrak{q}=a, b \sqsubseteq c$ holds iff at least one of the following is satisfied:

(1) $\mathfrak{p}=a, b \sqsubseteq c$,

(2) $\mathfrak{q}=a, b \sqsubseteq c$,

(3) $\mathfrak{p}=a, b \sqsubseteq x, \mathfrak{q}=y \sqsubseteq c$.

We leave the details of the argument to the reader.

Corollary 3.3. Assume that $\mathfrak{p}, \mathfrak{q}, \mathfrak{r}$ are as above, $\mathfrak{q} \in \mathbb{Q}_{\delta}$ and $\mathfrak{p} \leq \mathfrak{M}_{\delta}$. Then $\mathfrak{p} \oplus \mathfrak{q} \in \mathbb{Q}_{\delta}$ and $\mathfrak{p} \oplus_{x, y} \mathfrak{q} \in \mathbb{Q}_{\delta}$.

3.4. Definition. Let $(P, \sqsubseteq)$ be a partial order of size $\kappa, \kappa$ a regular cardinal. We say that $P$ satisfies the strong chain condition if whenever $\left(\mathfrak{X}_{\alpha}: \alpha<\kappa\right)$ is a sequence of finite suborderings of $(P, \sqsubseteq)$, and $x_{\alpha} \in X_{\alpha}$ for all $\alpha<\kappa$, then there are $\alpha<\beta<\kappa$ such that $\mathfrak{X}_{\alpha}$ and $\mathfrak{X}_{\beta}$ agree on $X_{\alpha} \cap X_{\beta}$, $\mathfrak{X}_{\alpha}$ and $\mathfrak{X}_{\beta}$ are separated end extensions of $\mathfrak{X}_{\alpha} \cap \mathfrak{X}_{\beta}, x_{\alpha}$ and $y_{\alpha}$ have the same type over $\mathfrak{X}_{\alpha} \cap \mathfrak{X}_{\beta}$, and

$$
\mathfrak{X}_{\alpha} \oplus_{x_{\alpha}, x_{\beta}} \mathfrak{X}_{\beta} \leq(P, \sqsubseteq) .
$$

3.5. Re mark. By the $\Delta$-system lemma, for any sequence $\left(X_{\alpha}: \alpha<\kappa\right)$ of finite sets there is a set $A \subseteq \kappa$ of size $\kappa$ such that the sets $\left(X_{\alpha}: \alpha \in A\right)$ form a $\Delta$-system. If $(P, \sqsubseteq)$ moreover satisfies $\mathrm{C} 3$, then we may additionally assume that for any $\alpha<\beta<\kappa$ the models $\mathfrak{X}_{\alpha}$ and $\mathfrak{X}_{\beta}$ are separated end extensions of $\mathfrak{X}_{\alpha} \cap \mathfrak{X}_{\beta}$. So the point of Definition 3.4 is really the last clause.

Lemma 3.6. Assume that $(P, \sqsubseteq)$ satisfies the strong chain condition. Then $(P, \sqsubseteq)$ satisfies conditions $\mathrm{C} 1$ and $\mathrm{C} 2$.

Pr o o f. To show C1, consider any family $\left(x_{\alpha}: \alpha<\kappa\right)$. Let $\mathfrak{X}_{\alpha}:=\left\{x_{\alpha}\right\}$. Since $(P, \sqsubseteq)$ satisfies the strong chain condition, we can find $\alpha<\beta$ such that $\mathfrak{X}_{\alpha} \oplus_{x_{\alpha}, x_{\beta}} \mathfrak{X}_{\beta} \leq(P, \sqsubseteq)$, but this means that $x_{\alpha} \sqsubseteq x_{\beta}$.

Finally, we show $\mathrm{C} 2$. Let $f:(P, \sqsubseteq) \rightarrow(P, \sqsubseteq)$ be monotone and assume that 1.1(C2) does not hold. This means that for all small sets $A$ there is $x$ such that $f(x) \neq x$ and $f(x) \notin A$. So we can find $\left(x_{\alpha}, y_{\alpha}: \alpha<\kappa\right)$ such that for all $\alpha, x_{\alpha} \neq f\left(x_{\alpha}\right)=y_{\alpha}$ and the sets $\left\{x_{\alpha}, y_{\alpha}\right\}$ are pairwise disjoint. Let $\mathfrak{X}_{\alpha}:=\left\{x_{\alpha}, y_{\alpha}\right\} \leq(P, \sqsubseteq)$. Without loss of generality, we either have $x_{\alpha} \sqsubseteq y_{\alpha}$ for all $\alpha$ or for no $\alpha$, similarly $y_{\alpha} \sqsubseteq x_{\alpha}$ for all $\alpha$ or for no $\alpha$. Now find $\alpha$ and $\beta$ such that $\mathfrak{X}_{\alpha} \oplus_{x_{\alpha}, x_{\beta}} \mathfrak{X}_{\beta} \leq \mathfrak{M}$. Now $\mathfrak{X}_{\alpha} \oplus_{x_{\alpha}, x_{\beta}} \mathfrak{X}_{\beta}=x_{\alpha} \sqsubseteq x_{\beta}$, but clearly $\mathfrak{X}_{\alpha} \oplus_{x_{\alpha}, x_{\beta}} \mathfrak{X}_{\beta}=y_{\alpha} \nsubseteq y_{\beta}$, so $f$ is not monotone, a contradiction. 
4. Forcing. In this section we will carry out the forcing construction that will prove the theorem.

We start with a universe $V_{0}$ satisfying GCH. Let $\kappa=\omega_{1}$, and let $\left(M_{\delta}\right.$ : $\delta \leq \kappa), A_{\alpha \beta}^{\delta}, \ldots, \Gamma_{\alpha \beta}^{\delta}$ be as in 2.4.

We define sequences $\left(\mathbb{P}_{\alpha}, \mathbb{Q}_{\alpha}: \alpha<\kappa\right),\left(\mathfrak{M}_{\alpha}: \alpha<\kappa\right),\left(\underset{\sim}{R_{\alpha}}: \alpha<\kappa\right)$ satisfying the following for all $\alpha<\kappa$ :

(1) $\mathbb{P}_{0}=\{\emptyset\}, \mathfrak{M}_{0}=\emptyset$.

(2) $\underset{\sim}{R}$ is a $\mathbb{P}_{\alpha}$-name of a subset of $M_{\alpha} \times M_{\alpha}$.

(3) In $V^{\mathbb{P}_{\alpha}}, F_{\alpha+1}$ is constructed from $R_{\alpha}$ as in 2.7-2.8.

(4) $\mathbb{Q}_{\alpha}$ is a $\mathbb{P}_{\alpha}$-name, $\Vdash_{\mathbb{P}_{\alpha}} \underset{\sim}{\mathbb{Q}}=\mathbb{Q}\left(\mathfrak{M}_{\alpha}, F_{\alpha+1}\right)$.

(5) $\widetilde{\mathbb{P}}_{\alpha+1}=\mathbb{P}_{\alpha} * \mathbb{Q}_{\alpha}$.

(6) $\mathbb{P}_{\alpha+1} \Vdash \mathfrak{M}_{\alpha+1}$ is the creature defined by the generic filter on $\mathbb{Q}_{\alpha}$, as in Section 2.

(7) If $\alpha$ is a limit, then $\mathbb{P}_{\alpha}$ is the finite support limit of $\left(\mathbb{P}_{\beta}: \beta<\alpha\right)$, and $\mathfrak{M}_{\alpha}=\bigcup_{\beta<\alpha} \mathfrak{M}_{\beta}$.

We let $\mathbb{P}_{\kappa}$ be the finite support limit of this iteration. Since all forcing notions involved satisfy the countable chain condition, we may (using the usual bookkeeping arguments) assume that:

$(*) \quad$ Whenever $\underset{\sim}{R}$ is a $\mathbb{P}_{\kappa}$-name of a subset of $M_{\kappa} \times M_{\kappa}$ of size $<\kappa$, then there is some $\alpha$ such that $\underset{\sim}{R}=\underset{\sim}{R}$.

Lemma 4.1. Let $A \subseteq M_{\delta}$ be finite. Define a set $\overline{\mathbb{P}}_{\delta}(A)$ as the set of all $\mathfrak{p} \in \mathbb{P}_{\delta}$ satisfying

$\exists \mathfrak{M}, \mathfrak{M}$ a finite creature, $A \subseteq M, \forall \alpha \in \operatorname{dom}(\mathfrak{p}): \mathfrak{p}\left\lceil\alpha \Vdash \mathfrak{p}(\alpha)=\mathfrak{M}\left\lceil M_{\alpha+1}\right.\right.$.

Then $\overline{\mathbb{P}}_{\delta}(A)$ is dense in $\mathbb{P}_{\delta}$. In particular, $\overline{\mathbb{P}}_{\delta}:=\overline{\mathbb{P}}_{\delta}(\emptyset)$ is dense in $\mathbb{P}_{\alpha}$.

Proof. By induction on $\delta$. Limit steps are easy.

Let $\mathfrak{p} \in \mathbb{P}_{\alpha+1}$. By strengthening $\mathfrak{p}\lceil\alpha$ we may assume that there is a creature $\mathfrak{N}$ such that $\mathfrak{p}\lceil\alpha \Vdash \mathfrak{p}(\alpha)=\mathfrak{N}$. By 2.10 we may assume that $A \subseteq N$. By inductive assumption we may assume that there is a creature $\mathfrak{M}$ witnessing $\mathfrak{p}\left\lceil\alpha \in \overline{\mathbb{P}}_{\alpha}\left(N \cap M_{\delta}\right)\right.$. Clearly $\mathfrak{M}$ and $\mathfrak{N}$ agree on $M \cap N$. Define $\mathfrak{M}^{\prime}:=\mathfrak{M} \oplus \mathfrak{N}$. So $\mathfrak{p}\left\lceil\alpha \Vdash \mathfrak{p}(\alpha) \leq \mathfrak{M}^{\prime}\right.$. Define $\mathfrak{p}^{\prime}$ by demanding $\mathfrak{p}^{\prime}\left\lceil\alpha=p\left\lceil\alpha\right.\right.$ and $\mathfrak{p}^{\prime}(\alpha)=\mathfrak{M}^{\prime}$. By 3.3, $\mathfrak{p}^{\prime}\left\lceil\alpha \Vdash \mathfrak{p}^{\prime}(\alpha) \in \mathbb{Q}_{\delta}\right.$. Clearly $\mathfrak{p}^{\prime} \geq \mathfrak{p}$, and $\mathfrak{p}^{\prime} \in \mathbb{P}_{\alpha+1}$.

In $V^{\mathbb{P}_{\kappa}}$, let $\mathfrak{M}_{\kappa}=\bigcup_{\alpha<\kappa} \mathfrak{M}_{\alpha}$. We claim that the structure $\left(\kappa, \sqsubseteq_{\mathfrak{M}_{\kappa}}\right)$ satisfies the four conditions from 1.1. The argument in Section 2 shows that we have $\mathrm{C} 3$ and $\mathrm{C} 4$. So, by 3.6 we only have to check that $\mathfrak{M}$ satisfies the strong chain condition.

Let $\underset{\sim}{X}:=\left(\underset{\sim}{X_{\alpha}}: \alpha<\kappa\right)$ be a sequence of names for finite creatures, and let $\left(x_{\alpha}: \alpha<\kappa\right)$ be forced to satisfy ${\underset{\sim}{\alpha}}_{\alpha} \in X_{\alpha}$. Assume that $\mathfrak{p}$ is a condition forcing that $\underset{\sim}{X}$ witnesses the failure of 3.4. For each $\alpha<\kappa$ we find $\mathfrak{p}_{\alpha} \geq \mathfrak{p}$ which decides $\mathfrak{X}_{\alpha}$ and $x_{\alpha}$. Let $e_{\alpha}=\operatorname{supp}\left(\mathfrak{p}_{\alpha}\right)$ and $\delta_{\alpha}:=\max \left(e_{\alpha}\right)+1$, so 
$\mathfrak{p}_{\alpha} \in \mathbb{P}_{\delta_{\alpha}}$. By 4.1 we may assume that there are finite creatures $\mathfrak{P}_{\alpha}$ such that for all $\varepsilon \in e_{\alpha}, p_{\alpha}\left\lceil\varepsilon \Vdash p(\varepsilon)=\mathfrak{P}_{\alpha}\left\lceil M_{\varepsilon+1}\right.\right.$. We may also assume that $X_{\alpha} \subseteq P_{\alpha}$, and hence (since $p_{\alpha} \Vdash \mathfrak{X}_{\alpha} \leq \mathfrak{M}_{\kappa}$ and $p_{\alpha} \Vdash \mathfrak{P}_{\alpha} \leq \mathfrak{M}_{\kappa}$ ), $\mathfrak{X}_{\alpha} \leq \mathfrak{P}_{\alpha}$.

We may assume that the creatures $\mathfrak{P}_{\alpha}$ form a $\Delta$-system, say with heart $\mathfrak{P}^{\Delta}$ (so in particular $\mathfrak{P}^{\Delta} \leq \mathfrak{P}_{\alpha}$ ). Moreover, we may assume that each $\mathfrak{P}_{\alpha}$ is a separated end extension of $\mathfrak{P}^{\Delta}$. Also, since the $x_{\alpha}$ are without loss of generality, all different, we may assume $x_{\alpha} \in P_{\alpha} \backslash P^{\Delta}$. Finally, we may assume that each $x_{\alpha}$ has the same type over $\mathfrak{P}^{\Delta}$.

Now pick any $\alpha<\beta$. We will find a condition $\mathfrak{q} \geq \mathfrak{p}_{\alpha}, \mathfrak{q} \geq \mathfrak{p}_{\beta}$ such that $\mathfrak{q} \Vdash \mathfrak{P}_{\alpha} \oplus_{x_{\alpha}, x_{\beta}} \mathfrak{P}_{\beta} \leq \mathfrak{M}_{\kappa}$.

Let $\mathfrak{Q}=\mathfrak{P}_{\alpha} \oplus_{x_{\alpha}, x_{\beta}} \mathfrak{P}_{\beta}$. First note that $\mathfrak{P}_{\alpha} \leq \mathfrak{Q}$ and $\mathfrak{P}_{\beta} \leq \mathfrak{Q}$ by 3.2. Let $\varepsilon^{*}$ be such that $x_{\beta} \in M_{\varepsilon^{*}+1} \backslash M_{\varepsilon}$.

Now note that

$$
\mathfrak{Q}\left\lceil M_{\varepsilon+1}= \begin{cases}\mathfrak{P}_{\alpha}\left\lceil M_{\varepsilon+1}\right. & \text { if } \varepsilon<\varepsilon^{*} \\ \mathfrak{P}_{\alpha} \oplus_{x_{\alpha}, x_{\beta}} \mathfrak{P}_{\beta}\left\lceil M_{\varepsilon+1}\right. & \text { if } \varepsilon \geq \varepsilon^{*} .\end{cases}\right.
$$

We now define a condition $\mathfrak{q}$ with $\operatorname{supp}(\mathfrak{q})=\operatorname{supp}\left(\mathfrak{p}_{\alpha}\right) \cup \operatorname{supp}\left(\mathfrak{p}_{\beta}\right)$, by stipulating $\mathfrak{q}(\varepsilon)=\mathfrak{Q}\left\lceil M_{\varepsilon+1}\right.$.

By 3.3 we know that $\mathfrak{q} \mid \varepsilon \Vdash \mathfrak{Q} \backslash M_{\varepsilon+1} \in \mathbb{Q}_{\varepsilon}$, so by induction it is clear that $\mathfrak{q}$ is indeed a condition. Clearly $\mathfrak{q} \Vdash \mathfrak{P}_{\alpha} \oplus_{x_{\alpha}, x_{\beta}} \mathfrak{P}_{\beta} \leq \mathfrak{M}_{\kappa}$.

\section{References}

[KS] H. Kaiser and N. Sauer, Order polynomially complete lattices, Algebra Universalis 130 (1993), 171-176.

[Sh 128] S. Shelah, Uncountable constructions for B.A., e.c. groups and Banach spaces, Israel J. Math. 51 (1985), 273-297.

[Sh 136] - Constructions of many complicated uncountable structures and Boolean algebras, ibid. 45 (1983), 100-146.

Technische Universität

Wiedner Hauptstrasse 8-10/118.2

A-1040 Wien, Austria

E-mail: Martin.Goldstern@tuwien.ac.at

Hebrew University of Jerusalem

Givat Ram

91094 Jerusalem, Israel

Current address:

Mathematik WE 2

Freie Universität

Arnimallee 3

D-14195 Berlin, Germany

E-mail: goldstrn@math.fu-berlin.de 